\title{
Barnes-type Daehee of the first kind and poly-Cauchy of the first kind mixed-type polynomials
}

\section{Dae San Kim', Taekyun Kim² ${ }^{2}$, Takao Komatsu ${ }^{3}$ and Sang-Hun Lee ${ }^{4}$}

*Correspondence: tkkim@kw.ac.kr ${ }^{2}$ Department of Mathematics, Kwangwoon University, Seoul, 139-701, Republic of Korea Full list of author information is available at the end of the article

\begin{abstract}
In this paper, by considering Barnes-type Daehee polynomials of the first kind as well as poly-Cauchy polynomials of the first kind, we define and investigate the mixed-type polynomials of these polynomials. From the properties of Sheffer sequences of these polynomials arising from umbral calculus, we derive new and interesting identities.

MSC: 05A15; 05A40; 11B68; 11B75; 65Q05
\end{abstract}

\section{Introduction}

In this paper, we consider the polynomials $D_{n}^{(k)}\left(x \mid a_{1}, \ldots, a_{r}\right)$ called the Barnes-type Daehee of the first kind and poly-Cauchy of the first kind mixed-type polynomials, whose generating function is given by

$$
\prod_{j=1}^{r}\left(\frac{\ln (1+t)}{(1+t)^{a_{j}}-1}\right) \operatorname{Lif}_{k}(\ln (1+t))(1+t)^{x}=\sum_{n=0}^{\infty} D_{n}^{(k)}\left(x \mid a_{1}, \ldots, a_{r}\right) \frac{t^{n}}{n !},
$$

where $a_{1}, \ldots, a_{r} \neq 0$. Here, $\operatorname{Lif}_{k}(x)(k \in \mathbb{Z})$ is the polyfactorial function [1] defined by

$$
\operatorname{Lif}_{k}(x)=\sum_{m=0}^{\infty} \frac{x^{m}}{m !(m+1)^{k}}
$$

When $x=0, D_{n}^{(k)}\left(a_{1}, \ldots, a_{r}\right)=D_{n}^{(k)}\left(0 \mid a_{1}, \ldots, a_{r}\right)$ is called Barnes-type Daehee of the first kind and poly-Cauchy of the first kind mixed-type number.

Recall that the Barnes-type Daehee polynomials of the first kind, denoted by $D_{n}\left(x \mid a_{1}\right.$, $\left.\ldots, a_{r}\right)$, are given by the generating function

$$
\prod_{j=1}^{r}\left(\frac{\ln (1+t)}{(1+t)^{a_{j}}-1}\right)(1+t)^{x}=\sum_{n=0}^{\infty} D_{n}\left(x \mid a_{1}, \ldots, a_{r}\right) \frac{t^{n}}{n !} .
$$

If $a_{1}=\cdots=a_{r}=1$, then $D_{n}^{(r)}(x)=D_{n}(x \mid \underbrace{1, \ldots, 1}_{r})$ are the Daehee polynomials of the first kind of order $r$. Dahee polynomials were defined by the second author [2] and have been investigated in $[3,4]$.

(2014 Kim et al.; licensee Springer. This is an Open Access article distributed under the terms of the Creative Commons Attribution License (http://creativecommons.org/licenses/by/2.0), which permits unrestricted use, distribution, and reproduction in any medium, provided the original work is properly cited. 
The poly-Cauchy polynomials of the first kind, denoted by $c_{n}^{(k)}(x)[5,6]$, are given by the generating function as

$$
\operatorname{Lif}_{k}(\ln (1+t))(1+t)^{x}=\sum_{n=0}^{\infty} c_{n}^{(k)}(-x) \frac{t^{n}}{n !}
$$

In this paper, by considering Barnes-type Daehee polynomials of the first kind as well as poly-Cauchy polynomials of the first kind, we define and investigate the mixed-type polynomials of these polynomials. From the properties of Sheffer sequences of these polynomials arising from umbral calculus, we derive new and interesting identities.

\section{Umbral calculus}

Let $\mathbb{C}$ be the complex number field and let $\mathcal{F}$ be the set of all formal power series in the variable $t$ :

$$
\mathcal{F}=\left\{f(t)=\sum_{k=0}^{\infty} \frac{a_{k}}{k !} t^{k} \mid a_{k} \in \mathbb{C}\right\} .
$$

Let $\mathbb{P}=\mathbb{C}[x]$ and let $\mathbb{P}^{*}$ be the vector space of all linear functionals on $\mathbb{P} .\langle L \mid p(x)\rangle$ is the action of the linear functional $L$ on the polynomial $p(x)$, and we recall that the vector space operations on $\mathbb{P}^{*}$ are defined by $\langle L+M \mid p(x)\rangle=\langle L \mid p(x)\rangle+\langle M \mid p(x)\rangle,\langle c L \mid p(x)\rangle=c\langle L \mid p(x)\rangle$, where $c$ is a complex constant in $\mathbb{C}$. For $f(t) \in \mathcal{F}$, let us define the linear functional on $\mathbb{P}$ by setting

$$
\left\langle f(t) \mid x^{n}\right\rangle=a_{n} \quad(n \geq 0) .
$$

In particular,

$$
\left\langle t^{k} \mid x^{n}\right\rangle=n ! \delta_{n, k} \quad(n, k \geq 0)
$$

where $\delta_{n, k}$ is the Kronecker symbol.

For $f_{L}(t)=\sum_{k=0}^{\infty} \frac{\left\langle L \mid x^{k}\right\rangle}{k !} t^{k}$, we have $\left\langle f_{L}(t) \mid x^{n}\right\rangle=\left\langle L \mid x^{n}\right\rangle$. That is, $L=f_{L}(t)$. The map $L \mapsto f_{L}(t)$ is a vector space isomorphism from $\mathbb{P}^{*}$ onto $\mathcal{F}$. Henceforth, $\mathcal{F}$ denotes both the algebra of formal power series in $t$ and the vector space of all linear functionals on $\mathbb{P}$, and so an element $f(t)$ of $\mathcal{F}$ will be thought of as both a formal power series and a linear functional. We call $\mathcal{F}$ the umbral algebra and the umbral calculus is the study of the umbral algebra. The order $O(f(t))$ of a power series $f(t)(\neq 0)$ is the smallest integer $k$ for which the coefficient of $t^{k}$ does not vanish. If $O(f(t))=1$, then $f(t)$ is called a delta series; if $O(f(t))=0$, then $f(t)$ is called an invertible series. For $f(t), g(t) \in \mathcal{F}$ with $O(f(t))=1$ and $O(g(t))=0$, there exists a unique sequence $s_{n}(x)\left(\operatorname{deg} s_{n}(x)=n\right)$ such that $\left\langle g(t) f(t)^{k} \mid s_{n}(x)\right\rangle=n ! \delta_{n, k}$, for $n, k \geq 0$. Such a sequence $s_{n}(x)$ is called the Sheffer sequence for $(g(t), f(t))$, which is denoted by $s_{n}(x) \sim(g(t), f(t))$.

For $f(t), g(t) \in \mathcal{F}$ and $p(x) \in \mathbb{P}$, we have

$$
\langle f(t) g(t) \mid p(x)\rangle=\langle f(t) \mid g(t) p(x)\rangle=\langle g(t) \mid f(t) p(x)\rangle
$$


and

$$
f(t)=\sum_{k=0}^{\infty}\left\langle f(t) \mid x^{k}\right\rangle \frac{t^{k}}{k !}, \quad p(x)=\sum_{k=0}^{\infty}\left\langle t^{k} \mid p(x)\right\rangle \frac{x^{k}}{k !}
$$

[7, Theorem 2.2.5]. Thus, by (6), we get

$$
t^{k} p(x)=p^{(k)}(x)=\frac{d^{k} p(x)}{d x^{k}} \quad \text { and } e^{y t} p(x)=p(x+y)
$$

Sheffer sequences are characterized by the generating function [7, Theorem 2.3.4].

Lemma 1 The sequence $s_{n}(x)$ is Sheffer for $(g(t), f(t))$ if and only if

$$
\frac{1}{g(\bar{f}(t))} e^{y \bar{f}(t)}=\sum_{k=0}^{\infty} \frac{s_{k}(y)}{k !} t^{k} \quad(y \in \mathbb{C}),
$$

where $\bar{f}(t)$ is the compositional inverse of $f(t)$.

For $s_{n}(x) \sim(g(t), f(t))$, we have the following equations [7, Theorem 2.3.7, Theorem 2.3.5, Theorem 2.3.9]:

$$
\begin{aligned}
& f(t) s_{n}(x)=n s_{n-1}(x) \quad(n \geq 0), \\
& s_{n}(x)=\sum_{j=0}^{n} \frac{1}{j !}\left\langle g(\bar{f}(t))^{-1} \bar{f}(t)^{j} \mid x^{n}\right\rangle x^{j}, \\
& s_{n}(x+y)=\sum_{j=0}^{n}\left(\begin{array}{l}
n \\
j
\end{array}\right) s_{j}(x) p_{n-j}(y),
\end{aligned}
$$

where $p_{n}(x)=g(t) s_{n}(x)$.

Assume that $p_{n}(x) \sim(1, f(t))$ and $q_{n}(x) \sim(1, g(t))$. Then the transfer formula [7, Corollary 3.8.2] is given by

$$
q_{n}(x)=x\left(\frac{f(t)}{g(t)}\right)^{n} x^{-1} p_{n}(x) \quad(n \geq 1) .
$$

For $s_{n}(x) \sim(g(t), f(t))$ and $r_{n}(x) \sim(h(t), l(t))$, assume that

$$
s_{n}(x)=\sum_{m=0}^{n} C_{n, m} r_{m}(x) \quad(n \geq 0) .
$$

Then we have [7, p.132]

$$
C_{n, m}=\frac{1}{m !}\left\langle\frac{h(\bar{f}(t))}{g(\bar{f}(t))} l(\bar{f}(t))^{m} \mid x^{n}\right\rangle .
$$




\section{Main results}

From the definition (1), $D_{n}^{(k)}\left(x \mid a_{1}, \ldots, a_{r}\right)$ is the Sheffer sequence for the pair

$$
g(t)=\prod_{j=1}^{r}\left(\frac{e^{a_{j} t}-1}{t}\right) \frac{1}{\operatorname{Lif}_{k}(t)} \quad \text { and } \quad f(t)=e^{t}-1 .
$$

So,

$$
D_{n}^{(k)}\left(x \mid a_{1}, \ldots, a_{r}\right) \sim\left(\prod_{j=1}^{r}\left(\frac{e^{a_{j} t}-1}{t}\right) \frac{1}{\operatorname{Lif}_{k}(t)}, e^{t}-1\right) .
$$

\subsection{Explicit expressions}

Recall that Barnes' multiple Bernoulli polynomials $B_{n}\left(x \mid a_{1}, \ldots, a_{r}\right)$ are defined by the generating function

$$
\frac{t^{r}}{\prod_{j=1}^{r}\left(e^{a_{j} t}-1\right)} e^{x t}=\sum_{n=0}^{\infty} B_{n}\left(x \mid a_{1}, \ldots, a_{r}\right) \frac{t^{n}}{n !},
$$

where $a_{1}, \ldots, a_{r} \neq 0[8,9]$. Let $(n)_{j}=n(n-1) \cdots(n-j+1)(j \geq 1)$ with $(n)_{0}=1$. The (signed) Stirling numbers of the first kind $S_{1}(n, m)$ are defined by

$$
(x)_{n}=\sum_{m=0}^{n} S_{1}(n, m) x^{m}
$$

\section{Theorem 1}

$$
\begin{aligned}
& D_{n}^{(k)}\left(x \mid a_{1}, \ldots, a_{r}\right) \\
& \quad=\sum_{m=0}^{n} \sum_{l=0}^{m} S_{1}(n, m) \frac{\left(\begin{array}{c}
m \\
l
\end{array}\right)}{(m-l+1)^{k}} B_{l}\left(x \mid a_{1}, \ldots, a_{r}\right) \\
& \quad=\sum_{j=0}^{n} \sum_{l=0}^{n-j} \sum_{i=0}^{l}\left(\begin{array}{l}
n \\
l
\end{array}\right)\left(\begin{array}{l}
l \\
i
\end{array}\right) S_{1}(n-l, j) c_{i}^{(k)} D_{l-i}\left(a_{1}, \ldots, a_{r}\right) x^{j} \\
& \quad=\sum_{l=0}^{n}\left(\begin{array}{l}
n \\
l
\end{array}\right) D_{n-l}\left(a_{1}, \ldots, a_{r}\right) c_{l}^{(k)}(-x) \\
& \quad=\sum_{l=0}^{n}\left(\begin{array}{l}
n \\
l
\end{array}\right) c_{n-l}^{(k)} D_{l}\left(x \mid a_{1}, \ldots, a_{r}\right) .
\end{aligned}
$$

Proof Since

$$
\prod_{j=1}^{r}\left(\frac{e^{a_{j} t}-1}{t}\right) \frac{1}{\operatorname{Lif}_{k}(t)} D_{n}^{(k)}\left(x \mid a_{1}, \ldots, a_{r}\right) \sim\left(1, e^{t}-1\right)
$$

and

$$
(x)_{n} \sim\left(1, e^{t}-1\right)
$$


we have

$$
\begin{aligned}
D_{n}^{(k)}\left(x \mid a_{1}, \ldots, a_{r}\right) & =\prod_{j=1}^{r}\left(\frac{t}{e^{a_{j} t}-1}\right) \operatorname{Lif}_{k}(t)(x)_{n} \\
& =\sum_{m=0}^{n} S_{1}(n, m) \prod_{j=1}^{r}\left(\frac{t}{e^{a_{j} t}-1}\right) \operatorname{Lif}_{k}(t) x^{m} \\
& =\sum_{m=0}^{n} S_{1}(n, m) \prod_{j=1}^{r}\left(\frac{t}{e^{a_{j} t}-1}\right) \sum_{l=0}^{m} \frac{t^{l}}{l !(l+1)^{k}} x^{m} \\
& =\sum_{m=0}^{n} S_{1}(n, m) \prod_{j=1}^{r}\left(\frac{t}{e^{a_{j} t}-1}\right) \sum_{l=0}^{m} \frac{(m)_{l}}{l !(l+1)^{k}} x^{m-l} \\
& =\sum_{m=0}^{n} S_{1}(n, m) \sum_{l=0}^{m} \frac{(m)_{l}}{l !(l+1)^{k}} \prod_{j=1}^{r}\left(\frac{t}{e^{a_{j} t}-1}\right) x^{m-l} \\
& =\sum_{m=0}^{n} \sum_{l=0}^{m} S_{1}(n, m) \frac{\left(\begin{array}{c}
m \\
l
\end{array}\right)}{(l+1)^{k}} B_{m-l} l\left(x \mid a_{1}, \ldots, a_{r}\right) \\
& =\sum_{m=0}^{n} \sum_{l=0}^{m} S_{1}(n, m) \frac{\left(\begin{array}{c}
m \\
l
\end{array}\right)}{(m-l+1)^{k}} B_{l}\left(x \mid a_{1}, \ldots, a_{r}\right) .
\end{aligned}
$$

Thus, we get (14).

By (9) with (12), we get

$$
\begin{aligned}
\left\langle g(\bar{f}(t))^{-1} \bar{f}(t)^{j} \mid x^{n}\right\rangle & \\
& =\left\langle\prod_{j=1}^{r}\left(\frac{\ln (1+t)}{(1+t)^{a_{j}}-1}\right) \operatorname{Lif}_{k}(\ln (1+t))(\ln (1+t))^{j} \mid x^{n}\right\rangle \\
& =\left\langle\prod_{j=1}^{r}\left(\frac{\ln (1+t)}{(1+t)^{a_{j}}-1}\right) \operatorname{Lif}_{k}(\ln (1+t)) \mid \sum_{l=0}^{\infty} \frac{j !}{(l+j) !} S_{1}(l+j, j) t^{l+j} x^{n}\right\rangle \\
& =\sum_{l=0}^{n-j} \frac{j !}{(l+j) !} S_{1}(l+j, j)(n)_{l+j}\left\langle\prod_{j=1}^{r}\left(\frac{\ln (1+t)}{(1+t)^{a_{j}}-1}\right) \operatorname{Lif}_{k}(\ln (1+t)) \mid x^{n-l-j}\right\rangle \\
& =\sum_{l=0}^{n-j} j !\left(\begin{array}{c}
n \\
l+j
\end{array}\right) S_{1}(l+j, j)\left\langle\sum_{i=0}^{\infty} D_{i}^{(k)}\left(a_{1}, \ldots, a_{r}\right) \frac{t^{i}}{i !} \mid x^{n-l-j}\right\rangle \\
& =\sum_{l=0}^{n-j} j !\left(\begin{array}{c}
n \\
l+j
\end{array}\right) S_{1}(l+j, j) D_{n-l-j}^{(k)}\left(a_{1}, \ldots, a_{r}\right) \\
& =\sum_{l=0}^{n-j} j !\left(\begin{array}{c}
n \\
l
\end{array}\right) S_{1}(n-l, j) D_{l}^{(k)}\left(a_{1}, \ldots, a_{r}\right) .
\end{aligned}
$$

On the other hand,

$$
\begin{aligned}
& \left\langle g(\bar{f}(t))^{-1} \bar{f}(t)^{j} \mid x^{n}\right\rangle \\
& \quad=\sum_{l=0}^{n-j} \frac{j !}{(l+j) !} S_{1}(l+j, j)(n)_{l+j}\left\langle\prod_{j=1}^{r}\left(\frac{\ln (1+t)}{(1+t)^{a_{j}}-1}\right) \mid \operatorname{Lif}_{k}(\ln (1+t)) x^{n-l-j}\right\rangle
\end{aligned}
$$




$$
\begin{aligned}
= & \sum_{l=0}^{n-j} j !\left(\begin{array}{c}
n \\
l+j
\end{array}\right) S_{1}(l+j, j)\left\langle\prod_{j=1}^{r}\left(\frac{\ln (1+t)}{(1+t)^{a_{j}}-1}\right) \mid \sum_{i=0}^{n-l-j} c_{i}^{(k)} \frac{t^{i}}{i !} x^{n-l-j}\right\rangle \\
= & \sum_{l=0}^{n-j} j !\left(\begin{array}{c}
n \\
l+j
\end{array}\right) S_{1}(l+j, j) \sum_{i=0}^{n-l-j} c_{i}^{(k)} \frac{(n-l-j)}{i !}\left\langle\prod_{j=1}^{r}\left(\frac{\ln (1+t)}{(1+t)^{a_{j}}-1}\right) \mid x^{n-l-j-i}\right\rangle \\
= & \sum_{l=0}^{n-j} j !\left(\begin{array}{c}
n \\
l+j
\end{array}\right) S_{1}(l+j, j) \sum_{i=0}^{n-l-j} c_{i}^{(k)} \frac{(n-l-j)_{i}}{i !}\left\langle\sum_{m=0}^{\infty} D_{m}\left(a_{1}, \ldots, a_{r}\right) \frac{t^{m}}{m !} \mid x^{n-l-j-i}\right\rangle \\
= & \sum_{l=0}^{n-j} \sum_{i=0}^{n-l-j} j !\left(\begin{array}{c}
n \\
l+j
\end{array}\right)\left(\begin{array}{c}
n-l-j \\
i
\end{array}\right) S_{1}(l+j, j) c_{i}^{(k)} D_{n-l-j-i}\left(a_{1}, \ldots, a_{r}\right) \\
= & \sum_{l=0}^{n-j} \sum_{i=0}^{l} j !\left(\begin{array}{c}
n \\
l
\end{array}\right)\left(\begin{array}{l}
l \\
i
\end{array}\right) S_{1}(n-l, j) c_{i}^{(k)} D_{l-i}\left(a_{1}, \ldots, a_{r}\right) .
\end{aligned}
$$

Thus, we obtain

$$
\begin{aligned}
D_{n}^{(k)} & \left(x \mid a_{1}, \ldots, a_{r}\right) \\
& =\sum_{j=0}^{n} \sum_{l=0}^{n-j}\left(\begin{array}{l}
n \\
l
\end{array}\right) S_{1}(n-l, j) D_{l}^{(k)}\left(a_{1}, \ldots, a_{r}\right) x^{j} \\
& =\sum_{j=0}^{n} \sum_{l=0}^{n-j} \sum_{i=0}^{l}\left(\begin{array}{l}
n \\
l
\end{array}\right)\left(\begin{array}{l}
l \\
i
\end{array}\right) S_{1}(n-l, j) c_{i}^{(k)} D_{l-i}\left(a_{1}, \ldots, a_{r}\right) x^{j},
\end{aligned}
$$

which is the identity (15).

Next,

$$
\begin{aligned}
D_{n}^{(k)}\left(y \mid a_{1}, \ldots, a_{r}\right) & =\left\langle\sum_{i=0}^{\infty} D_{i}^{(k)}\left(y \mid a_{1}, \ldots, a_{r}\right) \frac{t^{i}}{i !} \mid x^{n}\right\rangle \\
& =\left\langle\prod_{j=1}^{r}\left(\frac{\ln (1+t)}{(1+t)^{a_{j}}-1}\right) \operatorname{Lif}_{k}(\ln (1+t))(1+t)^{y} \mid x^{n}\right\rangle \\
& =\left\langle\prod_{j=1}^{r}\left(\frac{\ln (1+t)}{(1+t)^{a_{j}}-1}\right) \mid \operatorname{Lif}_{k}(\ln (1+t))(1+t)^{y} x^{n}\right\rangle \\
& =\left\langle\prod_{j=1}^{r}\left(\frac{\ln (1+t)}{(1+t)^{a_{j}}-1}\right) \mid \sum_{l=0}^{n} c_{l}^{(k)}(-y) \frac{t^{l}}{l !} x^{n}\right\rangle \\
& =\sum_{l=0}^{n}\left(\begin{array}{l}
n \\
l
\end{array}\right) c_{l}^{(k)}(-y)\left\langle\prod_{j=1}^{r}\left(\frac{\ln (1+t)}{(1+t)^{a_{j}}-1}\right) \mid x^{n-l}\right\rangle \\
& =\sum_{l=0}^{n}\left(\begin{array}{l}
n \\
l
\end{array}\right) c_{l}^{(k)}(-y)\left\langle\sum_{i=0}^{\infty} D_{i}\left(a_{1}, \ldots, a_{r}\right) \frac{t^{i}}{i !} \mid x^{n-l}\right\rangle \\
& =\sum_{l=0}^{n}\left(\begin{array}{l}
n \\
l
\end{array}\right) c_{l}^{(k)}(-y) D_{n-l}\left(a_{1}, \ldots, a_{r}\right) .
\end{aligned}
$$

Thus, we obtain (16). 
Finally, we obtain

$$
\begin{aligned}
D_{n}^{(k)}\left(y \mid a_{1}, \ldots, a_{r}\right) & =\left\langle\sum_{i=0}^{\infty} D_{i}^{(k)}\left(y \mid a_{1}, \ldots, a_{r}\right) \frac{t^{i}}{i !} \mid x^{n}\right\rangle \\
& =\left\langle\prod_{j=1}^{r}\left(\frac{\ln (1+t)}{(1+t)^{a_{j}}-1}\right) \operatorname{Lif}_{k}(\ln (1+t))(1+t)^{y} \mid x^{n}\right\rangle \\
& =\left\langle\operatorname{Lif}_{k}(\ln (1+t)) \mid \prod_{j=1}^{r}\left(\frac{\ln (1+t)}{(1+t)^{a_{j}}-1}\right)(1+t)^{y} x^{n}\right\rangle \\
& =\left\langle\operatorname{Lif}_{k}(\ln (1+t)) \mid \sum_{l=0}^{n} D_{l}\left(y \mid a_{1}, \ldots, a_{r}\right) \frac{t^{l}}{l !} x^{n}\right\rangle \\
& =\sum_{l=0}^{n} D_{l}\left(y \mid a_{1}, \ldots, a_{r}\right)\left(\begin{array}{l}
n \\
l
\end{array}\right)\left\langle\operatorname{Lif}_{k}(\ln (1+t)) \mid x^{n-l}\right\rangle \\
& =\sum_{l=0}^{n} D_{l}\left(y \mid a_{1}, \ldots, a_{r}\right)\left(\begin{array}{c}
n \\
l
\end{array}\right)\left\langle\sum_{i=0}^{\infty} c_{i}^{(k)} \frac{t^{i}}{i !} \mid x^{n-l}\right\rangle \\
& =\sum_{l=0}^{n}\left(\begin{array}{l}
n \\
l
\end{array}\right) D_{l}\left(y \mid a_{1}, \ldots, a_{r}\right) c_{n-l}^{(k)} .
\end{aligned}
$$

Thus, we get the identity (17).

\subsection{Sheffer identity}

\section{Theorem 2}

$$
D_{n}^{(k)}\left(x+y \mid a_{1}, \ldots, a_{r}\right)=\sum_{j=0}^{n}\left(\begin{array}{l}
n \\
j
\end{array}\right) D_{j}^{(k)}\left(x \mid a_{1}, \ldots, a_{r}\right)(y)_{n-j} .
$$

Proof By (12) with

$$
\begin{aligned}
p_{n}(x) & =\prod_{j=1}^{r}\left(\frac{e^{a_{j} t}-1}{t}\right) \frac{1}{\operatorname{Lif}_{k}(t)} D_{n}\left(x \mid a_{1}, \ldots, a_{r}\right) \\
& =(x)_{n} \sim\left(1, e^{t}-1\right),
\end{aligned}
$$

using (10), we have (20).

\subsection{Difference relations}

Theorem 3

$$
D_{n}^{(k)}\left(x+1 \mid a_{1}, \ldots, a_{r}\right)-D_{n}^{(k)}\left(x \mid a_{1}, \ldots, a_{r}\right)=n D_{n-1}^{(k)}\left(x \mid a_{1}, \ldots, a_{r}\right) .
$$

Proof By (8) with (12), we get

$$
\left(e^{t}-1\right) D_{n}^{(k)}\left(x \mid a_{1}, \ldots, a_{r}\right)=n D_{n-1}^{(k)}\left(x \mid a_{1}, \ldots, a_{r}\right) .
$$

By (7), we have (21). 


\subsection{Recurrence}

\section{Theorem 4}

$$
\begin{aligned}
D_{n+1}^{(k)}\left(x \mid a_{1}, \ldots, a_{r}\right)= & x D_{n}^{(k)}\left(x-1 \mid a_{1}, \ldots, a_{r}\right) \\
& -\sum_{m=0}^{n} \sum_{j=1}^{r} \sum_{l=1}^{m+1} \sum_{i=0}^{l} \frac{\left(\begin{array}{c}
m+1 \\
l
\end{array}\right)\left(\begin{array}{l}
l \\
i
\end{array}\right)}{(m+1)(l-i+1)^{k}} S_{1}(n, m) \\
& \times\left(-a_{j}\right)^{m+1-l} B_{m+1-l} B_{i}\left(x-1 \mid a_{1}, \ldots, a_{r}\right) \\
& +\sum_{m=0}^{n} \sum_{l=0}^{m} \frac{\left(\begin{array}{c}
m \\
l
\end{array}\right)}{(m+2-l)^{k}} S_{1}(n, m) B_{l}\left(x-1 \mid a_{1}, \ldots, a_{r}\right),
\end{aligned}
$$

where $B_{n}$ is the nth ordinary Bernoulli number.

Proof By applying

$$
s_{n+1}(x)=\left(x-\frac{g^{\prime}(t)}{g(t)}\right) \frac{1}{f^{\prime}(t)} s_{n}(x)
$$

[7, Corollary 3.7.2] with (12), we get

$$
D_{n+1}^{(k)}\left(x \mid a_{1}, \ldots, a_{r}\right)=x D_{n}^{(k)}\left(x-1 \mid a_{1}, \ldots, a_{r}\right)-e^{-t} \frac{g^{\prime}(t)}{g(t)} D_{n}^{(k)}\left(x \mid a_{1}, \ldots, a_{r}\right) .
$$

Now,

$$
\begin{aligned}
\frac{g^{\prime}(t)}{g(t)} & =(\ln g(t))^{\prime} \\
& =\left(\sum_{j=1}^{r} \ln \left(e^{a_{j} t}-1\right)-r \ln t-\ln \operatorname{Lif}_{k}(t)\right)^{\prime} \\
& =\sum_{j=1}^{r} \frac{a_{j} e^{a_{j} t}}{e^{a_{j} t}-1}-\frac{r}{t}-\frac{\operatorname{Lif}_{k}^{\prime}(t)}{\operatorname{Lif}_{k}(t)} \\
& =\frac{\sum_{j=1}^{r} \prod_{i \neq j}\left(e^{a_{i} t}-1\right)\left(a_{j} t e^{a_{j} t}-e^{a_{j} t}+1\right)}{t \prod_{j=1}^{r}\left(e^{a_{j} t}-1\right)}-\frac{\operatorname{Lif}_{k}^{\prime}(t)}{\operatorname{Lif}_{k}(t)} .
\end{aligned}
$$

Observe that

$$
\begin{aligned}
\frac{\sum_{j=1}^{r} \prod_{i \neq j}\left(e^{a_{i} t}-1\right)\left(a_{j} t e^{a_{j} t}-e^{a_{j} t}+1\right)}{\prod_{j=1}^{r}\left(e^{a_{j} t}-1\right)} \\
=\frac{\frac{1}{2}\left(\sum_{j=1}^{r} a_{1} \cdots a_{j-1} a_{j}^{2} a_{j+1} \cdots a_{r}\right) t^{r+1}+\cdots}{\left(a_{1} \cdots a_{r}\right) t^{r}+\cdots} \\
=\frac{1}{2}\left(\sum_{j=1}^{r} a_{j}\right) t+\cdots
\end{aligned}
$$


Kim et al. Advances in Difference Equations 2014, 2014:140

Page 9 of 22

is a series with order $\geq 1$. Since

$$
D_{n}^{(k)}\left(x \mid a_{1}, \ldots, a_{r}\right)=\prod_{j=1}^{r}\left(\frac{t}{e^{a_{j} t}-1}\right) \operatorname{Lif}_{k}(t)(x)_{n}=\sum_{m=0}^{n} S_{1}(n, m) \prod_{j=1}^{r}\left(\frac{t}{e^{a_{j} t}-1}\right) \operatorname{Lif}_{k}(t) x^{m}
$$

we have

$$
\begin{aligned}
\frac{g^{\prime}(t)}{g(t)} D_{n}^{(k)}\left(x \mid a_{1}, \ldots, a_{r}\right)= & \sum_{m=0}^{n} S_{1}(n, m) \frac{g^{\prime}(t)}{g(t)}\left(\prod_{j=1}^{r} \frac{t}{e^{a_{j} t}-1}\right) \operatorname{Lif}_{k}(t) x^{m} \\
= & \sum_{m=0}^{n} S_{1}(n, m) \operatorname{Lif}_{k}(t)\left(\prod_{j=1}^{r} \frac{t}{e^{a_{j} t}-1}\right) \\
& \times \frac{\sum_{j=1}^{r} \prod_{i \neq j}\left(e^{a_{i} t}-1\right)\left(a_{j} t e^{a_{j} t}-e^{a_{j} t}+1\right)}{t \prod_{j=1}^{r}\left(e^{a_{j} t}-1\right)} x^{m} \\
& -\sum_{m=0}^{n} S_{1}(n, m) \operatorname{Lif}_{k}^{\prime}(t)\left(\prod_{j=1}^{r} \frac{t}{e^{a_{j} t}-1}\right) x^{m} .
\end{aligned}
$$

Since

$$
\begin{aligned}
& \frac{\sum_{j=1}^{r} \prod_{i \neq j}\left(e^{a_{i} t}-1\right)\left(a_{j} t e^{a_{j} t}-e^{a_{j} t}+1\right)}{t \prod_{j=1}^{r}\left(e^{a_{j} t}-1\right)} x^{m} \\
& =\frac{\sum_{j=1}^{r} \prod_{i \neq j}\left(e^{a_{i} t}-1\right)\left(a_{j} t e^{a_{j} t}-e^{a_{j} t}+1\right)}{\prod_{j=1}^{r}\left(e^{a_{j} t}-1\right)} \frac{x^{m+1}}{m+1} \\
& =\frac{1}{m+1} \sum_{j=1}^{r}\left(\frac{a_{j} t e^{a_{j} t}}{e^{a_{j} t}-1}-1\right) x^{m+1} \\
& =\frac{1}{m+1} \sum_{j=1}^{r}\left(\sum_{l=0}^{\infty} \frac{(-1)^{l} B_{l} a_{j}^{l}}{l !} t^{l}-1\right) x^{m+1} \\
& =\frac{1}{m+1} \sum_{j=1}^{r}\left(\sum_{l=0}^{m+1}\left(\begin{array}{c}
m+1 \\
l
\end{array}\right)\left(-a_{j}\right)^{l} B_{l} x^{m+1-l}-x^{m+1}\right) \\
& =\frac{1}{m+1} \sum_{j=1}^{r} \sum_{l=1}^{m+1}\left(\begin{array}{c}
m+1 \\
l
\end{array}\right)\left(-a_{j}\right)^{l} B_{l} x^{m+1-l} \\
& \quad=\frac{1}{m+1} \sum_{j=1}^{r} \sum_{l=1}^{m+1}\left(\begin{array}{c}
m+1 \\
l
\end{array}\right)\left(-a_{j}\right)^{m+1-l} B_{m+1-l} x^{l},
\end{aligned}
$$

the first term in (24) is

$$
\begin{aligned}
& \sum_{m=0}^{n} \frac{S_{1}(n, m)}{m+1} \sum_{j=1}^{r} \sum_{l=1}^{m+1}\left(\begin{array}{c}
m+1 \\
l
\end{array}\right)\left(-a_{j}\right)^{m+1-l} B_{m+1-l} \operatorname{Lif}_{k}(t)\left(\prod_{j=1}^{r} \frac{t}{e^{a_{j} t}-1}\right) x^{l} \\
& \quad=\sum_{m=0}^{n} \frac{S_{1}(n, m)}{m+1} \sum_{j=1}^{r} \sum_{l=1}^{m+1}\left(\begin{array}{c}
m+1 \\
l
\end{array}\right)\left(-a_{j}\right)^{m+1-l} B_{m+1-l} \sum_{i=0}^{l} \frac{t^{i}}{i !(i+1)^{k}} B_{l}\left(x \mid a_{1}, \ldots, a_{r}\right)
\end{aligned}
$$




$$
\begin{aligned}
& =\sum_{m=0}^{n} \frac{S_{1}(n, m)}{m+1} \sum_{j=1}^{r} \sum_{l=1}^{m+1}\left(\begin{array}{c}
m+1 \\
l
\end{array}\right)\left(-a_{j}\right)^{m+1-l} B_{m+1-l} \sum_{i=0}^{l} \frac{\left(\begin{array}{l}
l \\
i
\end{array}\right)}{(i+1)^{k}} B_{l-i}\left(x \mid a_{1}, \ldots, a_{r}\right) \\
& =\sum_{m=0}^{n} \sum_{j=1}^{r} \sum_{l=1}^{m+1} \sum_{i=0}^{l} \frac{\left(\begin{array}{c}
m+1 \\
l
\end{array}\right)\left(\begin{array}{l}
l \\
i
\end{array}\right)}{(m+1)(l-i+1)^{k}} S_{1}(n, m)\left(-a_{j}\right)^{m+1-l} B_{m+1-l} B_{i}\left(x \mid a_{1}, \ldots, a_{r}\right) .
\end{aligned}
$$

Since

$$
\operatorname{Lif}_{k-1}(t)-\operatorname{Lif}_{k}(t)=\left(\frac{1}{2^{k-1}}-\frac{1}{2^{k}}\right) t+\cdots
$$

the second term in (24) is

$$
\begin{aligned}
\sum_{m=0}^{n} & S_{1}(n, m) \frac{\operatorname{Lif}_{k-1}(t)-\operatorname{Lif}_{k}(t)}{t} B_{m}\left(x \mid a_{1}, \ldots, a_{r}\right) \\
& =\sum_{m=0}^{n} S_{1}(n, m)\left(\operatorname{Lif}_{k-1}(t)-\operatorname{Lif}_{k}(t)\right) \frac{B_{m+1}\left(x \mid a_{1}, \ldots, a_{r}\right)}{m+1} \\
& =\sum_{m=0}^{n} \frac{S_{1}(n, m)}{m+1}\left(\operatorname{Lif}_{k-1}(t)-\operatorname{Lif}_{k}(t)\right) B_{m+1}\left(x \mid a_{1}, \ldots, a_{r}\right) \\
& =\sum_{m=0}^{n} \frac{S_{1}(n, m)}{m+1}\left(\sum_{l=0}^{m+1} \frac{t^{l}}{l !(l+1)^{k-1}} B_{m+1}\left(x \mid a_{1}, \ldots, a_{r}\right)-\sum_{l=0}^{m+1} \frac{t^{l}}{l !(l+1)^{k}} B_{m+1}\left(x \mid a_{1}, \ldots, a_{r}\right)\right) \\
& =\sum_{m=0}^{n} \frac{S_{1}(n, m)}{m+1}\left(\sum_{l=0}^{m+1} \frac{\left(\begin{array}{c}
m+1 \\
l
\end{array}\right)}{(l+1)^{k-1}} B_{m+1-l}\left(x \mid a_{1}, \ldots, a_{r}\right)-\sum_{l=0}^{m+1} \frac{\left(\begin{array}{c}
m+1 \\
l
\end{array}\right)}{(l+1)^{k}} B_{m+1-l}\left(x \mid a_{1}, \ldots, a_{r}\right)\right) \\
& =\sum_{m=0}^{n} \frac{S_{1}(n, m)}{m+1} \sum_{l=1}^{m+1} \frac{\left(\begin{array}{c}
m+1 \\
l
\end{array}\right) l}{(l+1)^{k}} B_{m+1-l}\left(x \mid a_{1}, \ldots, a_{r}\right) \\
& =\sum_{m=0}^{n} \sum_{l=1}^{m+1}\left(\begin{array}{c}
m \\
l-1
\end{array}\right) S_{1}(n, m) \frac{1}{(l+1)^{k}} B_{m+1-l}\left(x \mid a_{1}, \ldots, a_{r}\right) \\
& \sum_{m=0}^{m} \frac{\left(\begin{array}{c}
m \\
l
\end{array}\right)}{(m+2-l)^{k}} S_{1}(n, m) B_{l}\left(x \mid a_{1}, \ldots, a_{r}\right) .
\end{aligned}
$$

Thus, we have

$$
\begin{aligned}
D_{n+1}^{(k)}\left(x \mid a_{1}, \ldots, a_{r}\right)= & x D_{n}^{(k)}\left(x-1 \mid a_{1}, \ldots, a_{r}\right) \\
& -\sum_{m=0}^{n} \sum_{j=1}^{r} \sum_{l=1}^{m+1} \sum_{i=0}^{l} \frac{\left(\begin{array}{c}
m+1 \\
l
\end{array}\right)\left(\begin{array}{l}
l \\
i
\end{array}\right)}{(m+1)(l-i+1)^{k}} S_{1}(n, m) \\
& \times\left(-a_{j}\right)^{m+1-l} B_{m+1-l} B_{i}\left(x-1 \mid a_{1}, \ldots, a_{r}\right) \\
& +\sum_{m=0}^{n} \sum_{l=0}^{m} \frac{\left(\begin{array}{c}
m \\
l
\end{array}\right)}{(m+2-l)^{k}} S_{1}(n, m) B_{l}\left(x-1 \mid a_{1}, \ldots, a_{r}\right),
\end{aligned}
$$

which is the identity (22). 


\subsection{Differentiation}

\section{Theorem 5}

$$
\frac{d}{d x} D_{n}^{(k)}\left(x \mid a_{1}, \ldots, a_{r}\right)=n ! \sum_{l=0}^{n-1} \frac{(-1)^{n-l-1}}{l !(n-l)} D_{l}^{(k)}\left(x \mid a_{1}, \ldots, a_{r}\right)
$$

Proof We shall use

$$
\frac{d}{d x} s_{n}(x)=\sum_{l=0}^{n-1}\left(\begin{array}{l}
n \\
l
\end{array}\right)\left\langle\bar{f}(t) \mid x^{n-l}\right\rangle s_{l}(x)
$$

(cf. [7, Theorem 2.3.12]). Since

$$
\begin{aligned}
\left\langle\bar{f}(t) \mid x^{n-l}\right\rangle & =\left\langle\ln (1+t) \mid x^{n-l}\right\rangle \\
& =\left\langle\sum_{m=1}^{\infty} \frac{(-1)^{m-1} t^{m}}{m} \mid x^{n-l}\right\rangle \\
& =\sum_{m=1}^{n-l} \frac{(-1)^{m-1}}{m}\left\langle t^{m} \mid x^{n-l}\right\rangle \\
& =\sum_{m=1}^{n-l} \frac{(-1)^{m-1}}{m}(n-l) ! \delta_{m, n-l} \\
& =(-1)^{n-l-1}(n-l-1) !,
\end{aligned}
$$

with (12), we have

$$
\begin{aligned}
\frac{d}{d x} D_{n}^{(k)}\left(x \mid a_{1}, \ldots, a_{r}\right) & =\sum_{l=0}^{n-1}\left(\begin{array}{l}
n \\
l
\end{array}\right)(-1)^{n-l-1}(n-l-1) ! D_{l}^{(k)}\left(x \mid a_{1}, \ldots, a_{r}\right) \\
& =n ! \sum_{l=0}^{n-1} \frac{(-1)^{n-l-1}}{l !(n-l)} D_{l}^{(k)}\left(x \mid a_{1}, \ldots, a_{r}\right),
\end{aligned}
$$

which is the identity (26).

\subsection{One more relation}

The classical Cauchy numbers $c_{n}$ are defined by

$$
\frac{t}{\ln (1+t)}=\sum_{n=0}^{\infty} c_{n} \frac{t^{n}}{n !}
$$

(see e.g. $[1,10])$.

\section{Theorem 6}

$$
\begin{aligned}
D_{n}^{(k)}\left(x \mid a_{1}, \ldots, a_{r}\right)= & x D_{n-1}^{(k)}\left(x-1 \mid a_{1}, \ldots, a_{r}\right) \\
& +\frac{1}{n} \sum_{l=0}^{n}\left(\begin{array}{l}
n \\
l
\end{array}\right) c_{l} D_{n-l}^{(k-1)}\left(x-1 \mid a_{1}, \ldots, a_{r}\right)
\end{aligned}
$$




$$
\begin{aligned}
& +\frac{r-1}{n} \sum_{l=0}^{n}\left(\begin{array}{l}
n \\
l
\end{array}\right) c_{l} D_{n-l}^{(k)}\left(x-1 \mid a_{1}, \ldots, a_{r}\right) \\
& -\frac{1}{n} \sum_{j=1}^{r} \sum_{l=0}^{n}\left(\begin{array}{l}
n \\
l
\end{array}\right) a_{j} c_{l} D_{n-l}^{(k)}\left(x+a_{j}-1 \mid a_{1}, \ldots, a_{r}, a_{j}\right) .
\end{aligned}
$$

Proof For $n \geq 1$, we have

$$
\begin{aligned}
D_{n}^{(k)}\left(y \mid a_{1}, \ldots, a_{r}\right)= & \left\langle\sum_{l=0}^{\infty} D_{l}^{(k)}\left(y \mid a_{1}, \ldots, a_{r}\right) \frac{t^{l}}{l !} \mid x^{n}\right\rangle \\
= & \left\langle\prod_{j=1}^{r}\left(\frac{\ln (1+t)}{(1+t)^{a_{j}}-1}\right) \operatorname{Lif}_{k}(\ln (1+t))(1+t)^{y} \mid x^{n}\right\rangle \\
= & \left\langle\partial_{t}\left(\prod_{j=1}^{r}\left(\frac{\ln (1+t)}{(1+t)^{a_{j}}-1}\right) \operatorname{Lif}_{k}(\ln (1+t))(1+t)^{y}\right) \mid x^{n-1}\right\rangle \\
= & \left\langle\left(\partial_{t} \prod_{j=1}^{r}\left(\frac{\ln (1+t)}{(1+t)^{a_{j}}-1}\right)\right) \operatorname{Lif}_{k}(\ln (1+t))(1+t)^{y} \mid x^{n-1}\right\rangle \\
& +\left\langle\prod_{j=1}^{r}\left(\frac{\ln (1+t)}{(1+t)^{a_{j}}-1}\right)\left(\partial_{t} \operatorname{Lif}_{k}(\ln (1+t))\right)(1+t)^{y} \mid x^{n-1}\right\rangle \\
& +\left\langle\prod_{j=1}^{r}\left(\frac{\ln (1+t)}{(1+t)^{a_{j}}-1}\right) \operatorname{Lif}_{k}(\ln (1+t))\left(\partial_{t}(1+t)^{y}\right) \mid x^{n-1}\right\rangle .
\end{aligned}
$$

The third term is

$$
\begin{aligned}
& y\left\langle\prod_{j=1}^{r}\left(\frac{\ln (1+t)}{(1+t)^{a_{j}}-1}\right) \operatorname{Lif}_{k}(\ln (1+t))(1+t)^{y-1} \mid x^{n-1}\right\rangle \\
& \quad=y D_{n-1}^{(k)}\left(y-1 \mid a_{1}, \ldots, a_{r}\right) .
\end{aligned}
$$

By (25), the second term is

$$
\begin{aligned}
\left\langle\prod_{j=1}^{r}\left(\frac{\ln (1+t)}{(1+t)^{a_{j}}-1}\right) \frac{\operatorname{Lif}_{k-1}(\ln (1+t))-\operatorname{Lif}_{k}(\ln (1+t))}{(1+t) \ln (1+t)}(1+t)^{y} \mid x^{n-1}\right\rangle \\
=\left\langle\prod_{j=1}^{r}\left(\frac{\ln (1+t)}{(1+t)^{a_{j}}-1}\right) \frac{\operatorname{Lif}_{k-1}(\ln (1+t))-\operatorname{Lif}_{k}(\ln (1+t))}{t}(1+t)^{y-1} \mid \frac{t}{\ln (1+t)} x^{n-1}\right\rangle \\
=\left\langle\prod_{j=1}^{r}\left(\frac{\ln (1+t)}{(1+t)^{a_{j}}-1}\right) \frac{\operatorname{Lif}_{k-1}(\ln (1+t))-\operatorname{Lif}_{k}(\ln (1+t))}{t}(1+t)^{y-1} \mid \sum_{l=0}^{\infty} c_{l} \frac{t^{l}}{l !} x^{n-1}\right\rangle \\
=\sum_{l=0}^{n-1}\left(\begin{array}{c}
n-1 \\
l
\end{array}\right) c_{l} \quad \\
\quad \times\left\langle\prod_{j=1}^{r}\left(\frac{\ln (1+t)}{(1+t)^{a_{j}}-1}\right)(1+t)^{y-1} \mid \frac{\operatorname{Lif}_{k-1}(\ln (1+t))-\operatorname{Lif}_{k}(\ln (1+t))}{t} x^{n-1-l}\right\rangle
\end{aligned}
$$




$$
\begin{aligned}
= & \sum_{l=0}^{n-1}\left(\begin{array}{c}
n-1 \\
l
\end{array}\right) c_{l} \\
& \times\left\langle\prod_{j=1}^{r}\left(\frac{\ln (1+t)}{(1+t)^{a_{j}}-1}\right)(1+t)^{y-1} \mid\left(\operatorname{Lif}_{k-1}(\ln (1+t))-\operatorname{Lif}_{k}(\ln (1+t))\right) \frac{x^{n-l}}{n-l}\right\rangle \\
= & \frac{1}{n} \sum_{l=0}^{n-1}\left(\begin{array}{l}
n \\
l
\end{array}\right) c_{l}\left(\left\langle\prod_{j=1}^{r}\left(\frac{\ln (1+t)}{(1+t)^{a_{j}}-1}\right) \operatorname{Lif}_{k-1}(\ln (1+t))(1+t)^{y-1} \mid x^{n-l}\right\rangle\right. \\
& \left.-\left\langle\prod_{j=1}^{r}\left(\frac{\ln (1+t)}{(1+t)^{a_{j}}-1}\right) \operatorname{Lif}_{k}(\ln (1+t))(1+t)^{y-1} \mid x^{n-l}\right\rangle\right) \\
= & \frac{1}{n} \sum_{l=0}^{n-1}\left(\begin{array}{c}
n \\
l
\end{array}\right) c_{l}\left(D_{n-l}^{(k-1)}\left(y-1 \mid a_{1}, \ldots, a_{r}\right)-D_{n-l}^{(k)}\left(y-1 \mid a_{1}, \ldots, a_{r}\right)\right) .
\end{aligned}
$$

Since

$$
\partial_{t} \prod_{j=1}^{r}\left(\frac{\ln (1+t)}{(1+t)^{a_{j}}-1}\right)=\frac{1}{1+t} \prod_{j=1}^{r}\left(\frac{\ln (1+t)}{(1+t)^{a_{j}}-1}\right) \frac{\sum_{i=1}^{r}\left(\frac{t}{\ln (1+t)}-\frac{a_{i} t(1+t)^{a_{i}}}{(1+t)^{a_{i}}-1}\right)}{t}
$$

with

$$
\sum_{i=1}^{r}\left(\frac{t}{\ln (1+t)}-\frac{a_{i} t(1+t)^{a_{i}}}{(1+t)^{a_{i}}-1}\right)=-\frac{1}{2}\left(\sum_{i=1}^{r} a_{i}\right) t+\cdots
$$

a series with order $(\geq 1)$, the first term is

$$
\begin{aligned}
& \left\langle\prod_{j=1}^{r}\left(\frac{\ln (1+t)}{(1+t)^{a_{j}}-1}\right) \operatorname{Lif}_{k}(\ln (1+t))(1+t)^{y-1} \mid \frac{\sum_{i=1}^{r}\left(\frac{t}{\ln (1+t)}-\frac{a_{i} t(1+t)^{a_{i}}}{(1+t)^{a_{i}-1}}\right)}{t} x^{n-1}\right\rangle \\
& =\frac{1}{n}\left\langle\prod_{j=1}^{r}\left(\frac{\ln (1+t)}{(1+t)^{a_{j}}-1}\right) \operatorname{Lif}_{k}(\ln (1+t))(1+t)^{y-1} \mid \sum_{i=1}^{r}\left(\frac{t}{\ln (1+t)}-\frac{a_{i} t(1+t)^{a_{i}}}{(1+t)^{a_{i}}-1}\right) x^{n}\right\rangle \\
& =\frac{r}{n}\left\langle\prod_{j=1}^{r}\left(\frac{\ln (1+t)}{(1+t)^{a_{j}}-1}\right) \operatorname{Lif}_{k}(\ln (1+t))(1+t)^{y-1} \mid \frac{t}{\ln (1+t)} x^{n}\right\rangle \\
& -\frac{1}{n} \sum_{i=1}^{r} a_{i}\left\langle\frac{\ln (1+t)}{(1+t)^{a_{i}}-1} \prod_{j=1}^{r}\left(\frac{\ln (1+t)}{(1+t)^{a_{j}}-1}\right) \operatorname{Lif}_{k}(\ln (1+t))(1+t)^{y+a_{i}-1} \mid \frac{t}{\ln (1+t)} x^{n}\right\rangle \\
& =\frac{r}{n}\left\langle\prod_{j=1}^{r}\left(\frac{\ln (1+t)}{(1+t)^{a_{j}}-1}\right) \operatorname{Lif}_{k}(\ln (1+t))(1+t)^{y-1} \mid \sum_{l=0}^{\infty} c_{l} \frac{t^{l}}{l !} x^{n}\right\rangle \\
& -\frac{1}{n} \sum_{i=1}^{r} a_{i}\left\langle\frac{\ln (1+t)}{(1+t)^{a_{i}}-1} \prod_{j=1}^{r}\left(\frac{\ln (1+t)}{(1+t)^{a_{j}}-1}\right) \operatorname{Lif}_{k}(\ln (1+t))(1+t)^{y+a_{i}-1} \mid \sum_{l=0}^{\infty} c_{l} \frac{t^{l}}{l !} x^{n}\right\rangle \\
& =\frac{r}{n} \sum_{l=0}^{n}\left(\begin{array}{l}
n \\
l
\end{array}\right) c_{l}\left\langle\prod_{j=1}^{r}\left(\frac{\ln (1+t)}{(1+t)^{a_{j}}-1}\right) \operatorname{Lif}_{k}(\ln (1+t))(1+t)^{y-1} \mid x^{n-l}\right\rangle \\
& -\frac{1}{n} \sum_{i=1}^{r} a_{i} \sum_{l=0}^{n}\left(\begin{array}{l}
n \\
l
\end{array}\right) c_{l}
\end{aligned}
$$




$$
\begin{aligned}
& \times\left\langle\frac{\ln (1+t)}{(1+t)^{a_{i}}-1} \prod_{j=1}^{r}\left(\frac{\ln (1+t)}{(1+t)^{a_{j}}-1}\right) \operatorname{Lif}_{k}(\ln (1+t))(1+t)^{y+a_{i}-1} \mid x^{n-l}\right\rangle \\
= & \frac{r}{n} \sum_{l=0}^{n}\left(\begin{array}{c}
n \\
l
\end{array}\right) c_{l} D_{n-l}^{(k)}\left(y-1 \mid a_{1}, \ldots, a_{r}\right) \\
& -\frac{1}{n} \sum_{i=1}^{r} a_{i} \sum_{l=0}^{n}\left(\begin{array}{c}
n \\
l
\end{array}\right) c_{l} D_{n-l}^{(k)}\left(y+a_{i}-1 \mid a_{1}, \ldots, a_{r}, a_{i}\right) .
\end{aligned}
$$

Therefore, we obtain

$$
\begin{aligned}
& D_{n}^{(k)}\left(x \mid a_{1}, \ldots, a_{r}\right)=x D_{n-1}^{(k)}\left(x-1 \mid a_{1}, \ldots, a_{r}\right) \\
& +\frac{1}{n} \sum_{l=0}^{n-1}\left(\begin{array}{c}
n \\
l
\end{array}\right) c_{l}\left(D_{n-l}^{(k-1)}\left(x-1 \mid a_{1}, \ldots, a_{r}\right)-D_{n-l}^{(k)}\left(x-1 \mid a_{1}, \ldots, a_{r}\right)\right) \\
& +\frac{r}{n} \sum_{l=0}^{n}\left(\begin{array}{l}
n \\
l
\end{array}\right) c_{l} D_{n-l}^{(k)}\left(x-1 \mid a_{1}, \ldots, a_{r}\right) \\
& -\frac{1}{n} \sum_{j=1}^{r} \sum_{l=0}^{n}\left(\begin{array}{l}
n \\
l
\end{array}\right) a_{j} c_{l} D_{n-l}^{(k)}\left(x+a_{j}-1 \mid a_{1}, \ldots, a_{r}, a_{j}\right) \\
& =x D_{n-1}^{(k)}\left(x-1 \mid a_{1}, \ldots, a_{r}\right) \\
& +\frac{1}{n} \sum_{l=0}^{n-1}\left(\begin{array}{l}
n \\
l
\end{array}\right) c_{l} D_{n-l}^{(k-1)}\left(x-1 \mid a_{1}, \ldots, a_{r}\right) \\
& +\frac{r-1}{n} \sum_{l=0}^{n}\left(\begin{array}{l}
n \\
l
\end{array}\right) c_{l} D_{n-l}^{(k)}\left(x-1 \mid a_{1}, \ldots, a_{r}\right) \\
& +\frac{1}{n} c_{n}-\frac{1}{n} \sum_{j=1}^{r} \sum_{l=0}^{n}\left(\begin{array}{l}
n \\
l
\end{array}\right) a_{j} c_{l} D_{n-l}^{(k)}\left(x+a_{j}-1 \mid a_{1}, \ldots, a_{r}, a_{j}\right) \\
& =x D_{n-1}^{(k)}\left(x-1 \mid a_{1}, \ldots, a_{r}\right)+\frac{1}{n} \sum_{l=0}^{n}\left(\begin{array}{l}
n \\
l
\end{array}\right) c_{l} D_{n-l}^{(k-1)}\left(x-1 \mid a_{1}, \ldots, a_{r}\right) \\
& +\frac{r-1}{n} \sum_{l=0}^{n}\left(\begin{array}{l}
n \\
l
\end{array}\right) c_{l} D_{n-l}^{(k)}\left(x-1 \mid a_{1}, \ldots, a_{r}\right) \\
& -\frac{1}{n} \sum_{j=1}^{r} \sum_{l=0}^{n}\left(\begin{array}{l}
n \\
l
\end{array}\right) a_{j} c_{l} D_{n-l}^{(k)}\left(x+a_{j}-1 \mid a_{1}, \ldots, a_{r}, a_{j}\right),
\end{aligned}
$$

which is the identity (27).

\subsection{A relation including the Stirling numbers of the first kind}

Theorem 7 For $n \geq m \geq 1$, we have

$$
\begin{aligned}
& m \sum_{l=0}^{n-m}\left(\begin{array}{c}
n \\
l
\end{array}\right) S_{1}(n-l, m) D_{l}^{(k)}\left(a_{1}, \ldots, a_{r}\right) \\
& \quad=\frac{m r}{n} \sum_{l=0}^{n-m} \sum_{i=0}^{l}\left(\begin{array}{l}
n \\
l
\end{array}\right)\left(\begin{array}{l}
l \\
i
\end{array}\right) S_{1}(n-l, m) c_{l-i} D_{i}^{(k)}\left(-1 \mid a_{1}, \ldots, a_{r}\right)
\end{aligned}
$$




$$
\begin{aligned}
& -\frac{m}{n} \sum_{l=0}^{n-m} \sum_{i=0}^{l} \sum_{j=1}^{r}\left(\begin{array}{l}
n \\
l
\end{array}\right)\left(\begin{array}{l}
l \\
i
\end{array}\right) S_{1}(n-l, m) a_{j} c_{l-i} D_{i}^{(k)}\left(a_{j}-1 \mid a_{1}, \ldots, a_{r}, a_{j}\right) \\
& +\sum_{l=0}^{n-m}\left(\begin{array}{c}
n-1 \\
l
\end{array}\right) S_{1}(n-l-1, m-1) D_{l}^{(k-1)}\left(-1 \mid a_{1}, \ldots, a_{r}\right) \\
& +(m-1) \sum_{l=0}^{n-m}\left(\begin{array}{c}
n-1 \\
l
\end{array}\right) S_{1}(n-l-1, m-1) D_{l}^{(k)}\left(-1 \mid a_{1}, \ldots, a_{r}\right) .
\end{aligned}
$$

Proof We shall compute

$$
\left\langle\prod_{j=1}^{r}\left(\frac{\ln (1+t)}{(1+t)^{a_{j}}-1}\right) \operatorname{Lif}_{k}(\ln (1+t))(\ln (1+t))^{m} \mid x^{n}\right\rangle
$$

in two different ways. On the one hand,

$$
\begin{aligned}
\left\langle\prod_{j=1}^{r}\left(\frac{\ln (1+t)}{(1+t)^{a_{j}}-1}\right) \operatorname{Lif}_{k}(\ln (1+t))(\ln (1+t))^{m} \mid x^{n}\right\rangle \\
\quad=\left\langle\prod_{j=1}^{r}\left(\frac{\ln (1+t)}{(1+t)^{a_{j}}-1}\right) \operatorname{Lif}_{k}(\ln (1+t)) \mid(\ln (1+t))^{m} x^{n}\right\rangle \\
=\left\langle\prod_{j=1}^{r}\left(\frac{\ln (1+t)}{(1+t)^{a_{j}}-1}\right) \operatorname{Lif}_{k}(\ln (1+t)) \mid \sum_{l=0}^{\infty} \frac{m !}{(l+m) !} S_{1}(l+m, m) t^{l+m} x^{n}\right\rangle \\
=\sum_{l=0}^{n-m} \frac{m !}{(l+m) !} S_{1}(l+m, m)(n)_{l+m}\left\langle\prod_{j=1}^{r}\left(\frac{\ln (1+t)}{(1+t)^{a_{j}}-1}\right) \operatorname{Lif}_{k}(\ln (1+t)) \mid x^{n-l-m}\right\rangle \\
=\sum_{l=0}^{n-m} m !\left(\begin{array}{c}
n \\
l+m
\end{array}\right) S_{1}(l+m, m) D_{n-l-m}^{(k)}\left(a_{1}, \ldots, a_{r}\right) \\
=\sum_{l=0}^{n-m} m !\left(\begin{array}{c}
n \\
l
\end{array}\right) S_{1}(n-l, m) D_{l}^{(k)}\left(a_{1}, \ldots, a_{r}\right) .
\end{aligned}
$$

On the other hand,

$$
\begin{aligned}
\left\langle\prod_{j=1}^{r}\right. & \left(\frac{\ln (1+t)}{(1+t)^{a_{j}}-1}\right) \operatorname{Lif}_{k}(\ln (1+t))(\ln (1+t))^{m}\left|x^{n}\right\rangle \\
= & \left\langle\partial_{t}\left(\prod_{j=1}^{r}\left(\frac{\ln (1+t)}{(1+t)^{a_{j}}-1}\right) \operatorname{Lif}_{k}(\ln (1+t))(\ln (1+t))^{m}\right) \mid x^{n-1}\right\rangle \\
= & \left\langle\left(\partial_{t} \prod_{j=1}^{r}\left(\frac{\ln (1+t)}{(1+t)^{a_{j}}-1}\right)\right) \operatorname{Lif}_{k}(\ln (1+t))(\ln (1+t))^{m} \mid x^{n-1}\right\rangle \\
& +\left\langle\prod_{j=1}^{r}\left(\frac{\ln (1+t)}{(1+t)^{a_{j}}-1}\right)\left(\partial_{t} \operatorname{Lif}_{k}(\ln (1+t))\right)(\ln (1+t))^{m} \mid x^{n-1}\right\rangle \\
& +\left\langle\prod_{j=1}^{r}\left(\frac{\ln (1+t)}{(1+t)^{a_{j}}-1}\right) \operatorname{Lif}_{k}(\ln (1+t))\left(\partial_{t}(\ln (1+t))^{m}\right) \mid x^{n-1}\right\rangle
\end{aligned}
$$


The third term of (29) is equal to

$$
\begin{aligned}
& m\left\langle\prod_{j=1}^{r}\left(\frac{\ln (1+t)}{(1+t)^{a_{j}}-1}\right) \operatorname{Lif}_{k}(\ln (1+t))(1+t)^{-1} \mid(\ln (1+t))^{m-1} x^{n-1}\right\rangle \\
&=m\left\langle\prod_{j=1}^{r}\left(\frac{\ln (1+t)}{(1+t)^{a_{j}}-1}\right) \operatorname{Lif}_{k}(\ln (1+t))(1+t)^{-1}\right| \\
&\left.\quad \sum_{l=0}^{n-m} \frac{(m-1) !}{(l+m-1) !} S_{1}(l+m-1, m-1) t^{l+m-1} x^{n-1}\right\rangle \\
&=m \sum_{l=0}^{n-m} \frac{(m-1) !}{(l+m-1) !} S_{1}(l+m-1, m-1)(n-1)_{l+m-1} \\
& \quad \times\left\langle\prod_{j=1}^{r}\left(\frac{\ln (1+t)}{(1+t)^{a_{j}}-1}\right) \operatorname{Lif}_{k}(\ln (1+t))(1+t)^{-1} \mid x^{n-l-m}\right\rangle \\
&= m ! \sum_{l=0}^{n-m}\left(\begin{array}{c}
n-1 \\
l+m-1
\end{array}\right) S_{1}(l+m-1, m-1) D_{n-l-m}^{(k)}\left(-1 \mid a_{1}, \ldots, a_{r}\right) \\
&= m ! \sum_{l=0}^{n-m}\left(\begin{array}{c}
n-1 \\
l
\end{array}\right) S_{1}(n-l-1, m-1) D_{l}^{(k)}\left(-1 \mid a_{1}, \ldots, a_{r}\right) .
\end{aligned}
$$

The second term of (29) is equal to

$$
\begin{aligned}
\left\langle\prod_{j=1}^{r}\left(\frac{\ln (1+t)}{(1+t)^{a_{j}}-1}\right)\left(\frac{\operatorname{Lif}_{k-1}(\ln (1+t))-\operatorname{Lif}_{k}(\ln (1+t))}{(1+t) \ln (1+t)}\right)(\ln (1+t))^{m} \mid x^{n-1}\right\rangle \\
=\left\langle\prod_{j=1}^{r}\left(\frac{\ln (1+t)}{(1+t)^{a_{j}}-1}\right) \operatorname{Lif}_{k-1}(\ln (1+t))(1+t)^{-1} \mid(\ln (1+t))^{m-1} x^{n-1}\right\rangle \\
\quad-\left\langle\prod_{j=1}^{r}\left(\frac{\ln (1+t)}{(1+t)^{a_{j}}-1}\right) \operatorname{Lif}_{k}(\ln (1+t))(1+t)^{-1} \mid(\ln (1+t))^{m-1} x^{n-1}\right\rangle \\
=(m-1) ! \sum_{l=0}^{n-m}\left(\begin{array}{c}
n-1 \\
l
\end{array}\right) S_{1}(n-l-1, m-1) D_{l}^{(k-1)}\left(-1 \mid a_{1}, \ldots, a_{r}\right) \\
\quad-(m-1) ! \sum_{l=0}^{n-m}\left(\begin{array}{c}
n-1 \\
l
\end{array}\right) S_{1}(n-l-1, m-1) D_{l}^{(k)}\left(-1 \mid a_{1}, \ldots, a_{r}\right) .
\end{aligned}
$$

The first term of (29) is equal to

$$
\begin{aligned}
& \left\langle\frac{1}{1+t} \prod_{i=1}^{r}\left(\frac{\ln (1+t)}{(1+t)^{a_{i}}-1}\right) \frac{\sum_{j=1}^{r}\left(\frac{t}{\ln (1+t)}-\frac{a_{j} t(1+t)^{a_{j}}}{(1+t)^{a_{j}}-1}\right)}{t} \operatorname{Lif}_{k}(\ln (1+t))(\ln (1+t))^{m} \mid x^{n-1}\right\rangle \\
& =\left\langle\prod_{i=1}^{r}\left(\frac{\ln (1+t)}{(1+t)^{a_{i}}-1}\right) \operatorname{Lif}_{k}(\ln (1+t))(1+t)^{-1}(\ln (1+t))^{m}\right| \\
& \left.\quad \frac{\sum_{j=1}^{r}\left(\frac{t}{\ln (1+t)}-\frac{a_{j} t(1+t)^{a_{j}}}{(1+t)^{a_{j}}-1}\right)}{t} x^{n-1}\right\rangle
\end{aligned}
$$


Kim et al. Advances in Difference Equations 2014, 2014:140

Page 17 of 22

http://www.advancesindifferenceequations.com/content/2014/1/140

$$
\begin{aligned}
& =\frac{1}{n}\left\langle\prod_{i=1}^{r}\left(\frac{\ln (1+t)}{(1+t)^{a_{i}}-1}\right) \operatorname{Lif}_{k}(\ln (1+t))(1+t)^{-1}(\ln (1+t))^{m}\right| \\
& \left.\sum_{j=1}^{r}\left(\frac{t}{\ln (1+t)}-\frac{a_{j} t(1+t)^{a_{j}}}{(1+t)^{a_{j}}-1}\right) x^{n}\right\rangle \\
& =\frac{1}{n}\left\langle\prod_{i=1}^{r}\left(\frac{\ln (1+t)}{(1+t)^{a_{i}}-1}\right) \operatorname{Lif}_{k}(\ln (1+t))(1+t)^{-1}\right. \\
& \times \sum_{j=1}^{r}\left(\frac{t}{\ln (1+t)}-\frac{a_{j} t(1+t)^{a_{j}}}{(1+t)^{a_{j}}-1}\right)\left|(\ln (1+t))^{m} x^{n}\right\rangle \\
& =\frac{1}{n}\left\langle\prod_{i=1}^{r}\left(\frac{\ln (1+t)}{(1+t)^{a_{i}}-1}\right) \operatorname{Lif}_{k}(\ln (1+t))(1+t)^{-1}\right. \\
& \times \sum_{j=1}^{r}\left(\frac{t}{\ln (1+t)}-\frac{a_{j} t(1+t)^{a_{j}}}{(1+t)^{a_{j}}-1}\right)\left|\sum_{l=0}^{\infty} \frac{m !}{(l+m) !} S_{1}(l+m, m) t^{l+m} x^{n}\right\rangle \\
& =\frac{1}{n} \sum_{l=0}^{n-m} \frac{m !}{(l+m) !} S_{1}(l+m, m)(n)_{l+m}\left\langle\prod_{i=1}^{r}\left(\frac{\ln (1+t)}{(1+t)^{a_{i}}-1}\right) \operatorname{Lif}_{k}(\ln (1+t))(1+t)^{-1}\right. \\
& \times \sum_{j=1}^{r}\left(\frac{t}{\ln (1+t)}-\frac{a_{j} t(1+t)^{a_{j}}}{(1+t)^{a_{j}}-1}\right)\left|x^{n-l-m}\right\rangle \\
& =\frac{m !}{n} \sum_{l=0}^{n-m}\left(\begin{array}{c}
n \\
l+m
\end{array}\right) S_{1}(l+m, m) \\
& \times\left(r\left\langle\prod_{i=1}^{r}\left(\frac{\ln (1+t)}{(1+t)^{a_{i}}-1}\right) \operatorname{Lif}_{k}(\ln (1+t))(1+t)^{-1} \mid \frac{t}{\ln (1+t)} x^{n-l-m}\right\rangle\right. \\
& -\sum_{j=1}^{r} a_{j}\left\langle\frac{\ln (1+t)}{(1+t)^{a_{j}}-1} \prod_{i=1}^{r}\left(\frac{\ln (1+t)}{(1+t)^{a_{i}}-1}\right) \operatorname{Lif}_{k}(\ln (1+t))(1+t)^{a_{j}-1}\right| \\
& \left.\left.\frac{t}{\ln (1+t)} x^{n-l-m}\right\rangle\right) \\
& =\frac{m !}{n} \sum_{l=0}^{n-m}\left(\begin{array}{c}
n \\
l+m
\end{array}\right) S_{1}(l+m, m) \\
& \times\left\langle r\left\langle\prod_{i=1}^{r}\left(\frac{\ln (1+t)}{(1+t)^{a_{i}}-1}\right) \operatorname{Lif}_{k}(\ln (1+t))(1+t)^{-1} \mid \sum_{\nu=0}^{\infty} c_{\nu} \frac{t^{v}}{\nu !} x^{n-l-m}\right\rangle\right. \\
& -\sum_{j=1}^{r} a_{j}\left\langle\frac{\ln (1+t)}{(1+t)^{a_{j}}-1} \prod_{i=1}^{r}\left(\frac{\ln (1+t)}{(1+t)^{a_{i}}-1}\right) \operatorname{Lif}_{k}(\ln (1+t))(1+t)^{a_{j}-1}\right| \\
& \left.\left.\sum_{\nu=0}^{\infty} c_{\nu} \frac{t^{\nu}}{\nu !} x^{n-l-m}\right\rangle\right) \\
& =\frac{m !}{n} \sum_{l=0}^{n-m}\left(\begin{array}{c}
n \\
l+m
\end{array}\right) S_{1}(l+m, m)
\end{aligned}
$$




$$
\begin{aligned}
& \times\left(r \sum_{\nu=0}^{n-l-m}\left(\begin{array}{c}
n-l-m \\
v
\end{array}\right) c_{\nu}\left\langle\prod_{i=1}^{r}\left(\frac{\ln (1+t)}{(1+t)^{a_{i}}-1}\right) \operatorname{Lif}_{k}(\ln (1+t))(1+t)^{-1} \mid x^{n-l-m-\nu}\right\rangle\right. \\
& -\sum_{j=1}^{r} a_{j} \sum_{\nu=0}^{n-l-m}\left(\begin{array}{c}
n-l-m \\
v
\end{array}\right) c_{v} \\
& \left.\times\left\langle\frac{\ln (1+t)}{(1+t)^{a_{j}}-1} \prod_{i=1}^{r}\left(\frac{\ln (1+t)}{(1+t)^{a_{i}}-1}\right) \operatorname{Lif}_{k}(\ln (1+t))(1+t)^{a_{j}-1} \mid x^{n-l-m-v}\right\rangle\right) \\
& =\frac{m !}{n} \sum_{l=0}^{n-m}\left(\begin{array}{c}
n \\
l+m
\end{array}\right) S_{1}(l+m, m) \\
& \times\left(r \sum_{v=0}^{n-l-m}\left(\begin{array}{c}
n-l-m \\
v
\end{array}\right) c_{\nu} D_{n-l-m-v}^{(k)}\left(-1 \mid a_{1}, \ldots, a_{r}\right)\right. \\
& \left.-\sum_{j=1}^{r} \sum_{v=0}^{n-l-m}\left(\begin{array}{c}
n-l-m \\
v
\end{array}\right) a_{j} c_{v} D_{n-l-m-v}^{(k)}\left(a_{j}-1 \mid a_{1}, \ldots, a_{r}, a_{j}\right)\right) \\
& =\frac{m !}{n} \sum_{l=0}^{n-m}\left(\begin{array}{l}
n \\
l
\end{array}\right) S_{1}(n-l, m) \\
& \times\left(r \sum_{i=0}^{l}\left(\begin{array}{l}
l \\
i
\end{array}\right) c_{i} D_{n-i}^{(k)}\left(-1 \mid a_{1}, \ldots, a_{r}\right)-\sum_{j=1}^{r} \sum_{i=0}^{l}\left(\begin{array}{l}
l \\
i
\end{array}\right) a_{j} c_{i} D_{l-i}^{(k)}\left(a_{j}-1 \mid a_{1}, \ldots, a_{r}, a_{j}\right)\right) .
\end{aligned}
$$

Therefore, we get for $n \geq m \geq 1$

$$
\begin{aligned}
& m ! \sum_{l=0}^{n-m}\left(\begin{array}{c}
n \\
l
\end{array}\right) S_{1}(n-l, m) D_{l}^{(k)}\left(a_{1}, \ldots, a_{r}\right) \\
& =m ! \frac{r}{n} \sum_{l=0}^{n-m} \sum_{i=0}^{l}\left(\begin{array}{l}
n \\
l
\end{array}\right)\left(\begin{array}{l}
l \\
i
\end{array}\right) S_{1}(n-l, m) c_{i} D_{l-i}^{(k)}\left(-1 \mid a_{1}, \ldots, a_{r}\right) \\
& \quad-m ! \frac{1}{n} \sum_{l=0}^{n-m} \sum_{i=0}^{l} \sum_{j=1}^{r}\left(\begin{array}{l}
n \\
l
\end{array}\right)\left(\begin{array}{l}
l \\
i
\end{array}\right) S_{1}(n-l, m) a_{j} c_{i} D_{l-i}^{(k)}\left(a_{j}-1 \mid a_{1}, \ldots, a_{r}, a_{j}\right) \\
& \quad+(m-1) ! \sum_{l=0}^{n-m}\left(\begin{array}{c}
n-1 \\
l
\end{array}\right) S_{1}(n-l-1, m-1) D_{l}^{(k-1)}\left(-1 \mid a_{1}, \ldots, a_{r}\right) \\
& \quad-(m-1) ! \sum_{l=0}^{n-m}\left(\begin{array}{c}
n-1 \\
l
\end{array}\right) S_{1}(n-l-1, m-1) D_{l}^{(k)}\left(-1 \mid a_{1}, \ldots, a_{r}\right) \\
& \quad+m ! \sum_{l=0}^{n-m}\left(\begin{array}{c}
n-1 \\
l
\end{array}\right) S_{1}(n-l-1, m-1) D_{l}^{(k)}\left(-1 \mid a_{1}, \ldots, a_{r}\right) .
\end{aligned}
$$

Dividing both sides by $(m-1)$ !, we obtain for $n \geq m \geq 1$

$$
\begin{aligned}
& m \sum_{l=0}^{n-m}\left(\begin{array}{l}
n \\
l
\end{array}\right) S_{1}(n-l, m) D_{l}^{(k)}\left(a_{1}, \ldots, a_{r}\right) \\
& \quad=\frac{m r}{n} \sum_{l=0}^{n-m} \sum_{i=0}^{l}\left(\begin{array}{l}
n \\
l
\end{array}\right)\left(\begin{array}{l}
l \\
i
\end{array}\right) S_{1}(n-l, m) c_{l-i} D_{i}^{(k)}\left(-1 \mid a_{1}, \ldots, a_{r}\right)
\end{aligned}
$$




$$
\begin{aligned}
& -\frac{m}{n} \sum_{l=0}^{n-m} \sum_{i=0}^{l} \sum_{j=1}^{r}\left(\begin{array}{l}
n \\
l
\end{array}\right)\left(\begin{array}{l}
l \\
i
\end{array}\right) S_{1}(n-l, m) a_{j} c_{l-i} D_{i}^{(k)}\left(a_{j}-1 \mid a_{1}, \ldots, a_{r}, a_{j}\right) \\
& +\sum_{l=0}^{n-m}\left(\begin{array}{c}
n-1 \\
l
\end{array}\right) S_{1}(n-l-1, m-1) D_{l}^{(k-1)}\left(-1 \mid a_{1}, \ldots, a_{r}\right) \\
& +(m-1) \sum_{l=0}^{n-m}\left(\begin{array}{c}
n-1 \\
l
\end{array}\right) S_{1}(n-l-1, m-1) D_{l}^{(k)}\left(-1 \mid a_{1}, \ldots, a_{r}\right) .
\end{aligned}
$$

Thus, we get (28).

\subsection{A relation with the falling factorials}

Theorem 8

$$
D_{n}^{(k)}\left(x \mid a_{1}, \ldots, a_{r}\right)=\sum_{m=0}^{n}\left(\begin{array}{c}
n \\
m
\end{array}\right) D_{n-m}^{(k)}\left(a_{1}, \ldots, a_{r}\right)(x)_{m} .
$$

Proof For (12) and (19), assume that $D_{n}^{(k)}\left(x \mid a_{1}, \ldots, a_{r}\right)=\sum_{m=0}^{n} C_{n, m}(x)_{m}$. By (11), we have

$$
\begin{aligned}
C_{n, m} & =\frac{1}{m !}\left\langle\frac{1}{\prod_{j=1}^{r}\left(\frac{e^{a_{j} \ln (1+t)}-1}{\ln (1+t)}\right) \frac{1}{\operatorname{Lif}_{k}(\ln (1+t))}} t^{m} \mid x^{n}\right\rangle \\
& =\frac{1}{m !}\left\langle\prod_{j=1}^{r}\left(\frac{\ln (1+t)}{(1+t)^{a_{j}}-1}\right) \operatorname{Lif}_{k}(\ln (1+t)) \mid t^{m} x^{n}\right\rangle \\
& =\left(\begin{array}{c}
n \\
m
\end{array}\right)\left\langle\prod_{j=1}^{r}\left(\frac{\ln (1+t)}{(1+t)^{a_{j}}-1}\right) \operatorname{Lif}_{k}(\ln (1+t)) \mid x^{n-m}\right\rangle \\
& =\left(\begin{array}{c}
n \\
m
\end{array}\right) D_{n-m}^{(k)}\left(a_{1}, \ldots, a_{r}\right) .
\end{aligned}
$$

Thus, we get the identity (30).

\subsection{A relation with higher-order Frobenius-Euler polynomials}

For $\lambda \in \mathbb{C}$ with $\lambda \neq 1$, the Frobenius-Euler polynomials of order $r, H_{n}^{(r)}(x \mid \lambda)$ are defined by the generating function

$$
\left(\frac{1-\lambda}{e^{t}-\lambda}\right)^{r} e^{x t}=\sum_{n=0}^{\infty} H_{n}^{(r)}(x \mid \lambda) \frac{t^{n}}{n !}
$$

(see e.g. [11]).

\section{Theorem 9}

$$
\begin{aligned}
D_{n}^{(k)}\left(x \mid a_{1}, \ldots, a_{r}\right)= & \sum_{m=0}^{n}\left(\sum_{j=0}^{n-m} \sum_{l=0}^{n-m-j}\left(\begin{array}{c}
s \\
j
\end{array}\right)\left(\begin{array}{c}
n-j \\
l
\end{array}\right)(n)_{j}\right. \\
& \left.\times(1-\lambda)^{-j} S_{1}(n-j-l, m) D_{l}^{(k)}\left(a_{1}, \ldots, a_{r}\right)\right) H_{m}^{(s)}(x \mid \lambda) .
\end{aligned}
$$


Proof For (12) and

$$
H_{n}^{(s)}(x \mid \lambda) \sim\left(\left(\frac{e^{t}-\lambda}{1-\lambda}\right)^{s}, t\right),
$$

assume that $D_{n}^{(k)}\left(x \mid a_{1}, \ldots, a_{r}\right)=\sum_{m=0}^{n} C_{n, m} H_{m}^{(s)}(x \mid \lambda)$. By (11), similarly to the proof of (28), we have

$$
\begin{aligned}
& C_{n, m}=\frac{1}{m !}\left\langle\frac{\left(\frac{e^{\ln (1+t)}-\lambda}{1-\lambda}\right)^{s}}{\prod_{j=1}^{r}\left(\frac{e^{a_{j} \ln (1+t)}-1}{\ln (1+t)}\right) \frac{1}{\operatorname{Lif}_{k}(\ln (1+t))}}(\ln (1+t))^{m} \mid x^{n}\right\rangle \\
& =\frac{1}{m !(1-\lambda)^{s}}\left\langle\prod_{j=1}^{r}\left(\frac{\ln (1+t)}{(1+t)^{a_{j}}-1}\right) \operatorname{Lif}_{k}(\ln (1+t))(\ln (1+t))^{m}(1-\lambda+t)^{s} \mid x^{n}\right\rangle \\
& =\frac{1}{m !(1-\lambda)^{s}} \\
& \times\left\langle\prod_{j=1}^{r}\left(\frac{\ln (1+t)}{(1+t)^{a_{j}}-1}\right) \operatorname{Lif}_{k}(\ln (1+t))(\ln (1+t))^{m} \mid \sum_{i=0}^{\min \{s, n\}}\left(\begin{array}{l}
s \\
i
\end{array}\right)(1-\lambda)^{s-i} t^{i} x^{n}\right\rangle \\
& =\frac{1}{m !(1-\lambda)^{s}} \sum_{i=0}^{n-m}\left(\begin{array}{l}
s \\
i
\end{array}\right)(1-\lambda)^{s-i}(n)_{i} \\
& \times\left\langle\prod_{j=1}^{r}\left(\frac{\ln (1+t)}{(1+t)^{a_{j}}-1}\right) \operatorname{Lif}_{k}(\ln (1+t))(\ln (1+t))^{m} \mid x^{n-i}\right\rangle \\
& =\frac{1}{m !(1-\lambda)^{s}} \sum_{i=0}^{n-m}\left(\begin{array}{l}
s \\
i
\end{array}\right)(1-\lambda)^{s-i}(n)_{i} \sum_{l=0}^{n-m-i} m !\left(\begin{array}{c}
n-i \\
l
\end{array}\right) S_{1}(n-i-l, m) D_{l}^{(k)}\left(a_{1}, \ldots, a_{r}\right) \\
& =\sum_{i=0}^{n-m} \sum_{l=0}^{n-m-i}\left(\begin{array}{c}
s \\
i
\end{array}\right)\left(\begin{array}{c}
n-i \\
l
\end{array}\right)(n)_{i}(1-\lambda)^{-i} S_{1}(n-i-l, m) D_{l}^{(k)}\left(a_{1}, \ldots, a_{r}\right) .
\end{aligned}
$$

Thus, we get the identity (31).

\subsection{A relation with higher-order Bernoulli polynomials}

Bernoulli polynomials $\mathfrak{B}_{n}^{(r)}(x)$ of order $r$ are defined by

$$
\left(\frac{t}{e^{t}-1}\right)^{r} e^{x t}=\sum_{n=0}^{\infty} \frac{\mathfrak{B}_{n}^{(r)}(x)}{n !} t^{n}
$$

(see e.g. [7, Section 2.2]). In addition, Cauchy numbers of the first kind $\mathfrak{C}_{n}^{(r)}$ of order $r$ are defined by

$$
\left(\frac{t}{\ln (1+t)}\right)^{r}=\sum_{n=0}^{\infty} \frac{\mathfrak{C}_{n}^{(r)}}{n !} t^{n}
$$

(see e.g. [12, (2.1)], [13, (6)]). 


\section{Theorem 10}

$$
\begin{aligned}
& D_{n}^{(k)}\left(x \mid a_{1}, \ldots, a_{r}\right) \\
& \quad=\sum_{m=0}^{n}\left(\sum_{i=0}^{n-m} \sum_{l=0}^{n-m-i}\left(\begin{array}{c}
n \\
i
\end{array}\right)\left(\begin{array}{c}
n-i \\
l
\end{array}\right) \mathfrak{C}_{i}^{(s)} S_{1}(n-i-l, m) D_{l}^{(k)}\left(a_{1}, \ldots, a_{r}\right)\right) \mathfrak{B}_{m}^{(s)}(x) .
\end{aligned}
$$

Proof For (12) and

$$
\mathfrak{B}_{n}^{(s)}(x) \sim\left(\left(\frac{e^{t}-1}{t}\right)^{s}, t\right),
$$

assume that $D_{n}^{(k)}\left(x \mid a_{1}, \ldots, a_{r}\right)=\sum_{m=0}^{n} C_{n, m} \mathfrak{B}_{m}^{(s)}(x)$. By (11), similarly to the proof of (28), we have

$$
\begin{aligned}
C_{n, m} & =\frac{1}{m !}\left\langle\frac{\left(\frac{\ln ^{\ln (1+t)}-1}{\ln (1+t)}\right)^{s}}{\prod_{j=1}^{r}\left(\frac{e^{a_{j} \ln (1+t)}-1}{\ln (1+t)}\right) \frac{1}{\operatorname{Lif}_{k}(\ln (1+t))}}(\ln (1+t))^{m} \mid x^{n}\right\rangle \\
& =\frac{1}{m !}\left\langle\prod_{j=1}^{r}\left(\frac{\ln (1+t)}{(1+t)^{a_{j}}-1}\right) \operatorname{Lif}_{k}(\ln (1+t))(\ln (1+t))^{m} \mid\left(\frac{t}{\ln (1+t)}\right)^{s} x^{n}\right\rangle \\
& =\frac{1}{m !}\left\langle\prod_{j=1}^{r}\left(\frac{\ln (1+t)}{(1+t)^{a_{j}}-1}\right) \operatorname{Lif}_{k}(\ln (1+t))(\ln (1+t))^{m} \mid \sum_{i=0}^{\infty} \mathfrak{C}_{i}^{(s)} \frac{t^{i}}{i !} x^{n}\right\rangle \\
& =\frac{1}{m !} \sum_{i=0}^{n-m} \mathfrak{C}_{i}^{(s)}\left(\begin{array}{c}
n \\
i
\end{array}\right)\left\langle\prod_{j=1}^{r}\left(\frac{\ln (1+t)}{(1+t)^{a_{j}}-1}\right) \operatorname{Lif}_{k}(\ln (1+t))(\ln (1+t))^{m} \mid x^{n-i}\right\rangle \\
& =\frac{1}{m !} \sum_{i=0}^{n-m} \mathfrak{C}_{i}^{(s)}\left(\begin{array}{c}
n \\
i
\end{array}\right) \sum_{l=0}^{n-m-i} m !\left(\begin{array}{c}
n-i \\
l
\end{array}\right) S_{1}(n-i-l, m) D_{l}^{(k)}\left(a_{1}, \ldots, a_{r}\right) \\
& =\sum_{i=0}^{n-m} \sum_{l=0}^{n-m-i}\left(\begin{array}{c}
n \\
i
\end{array}\right)\left(\begin{array}{c}
n-i \\
l
\end{array}\right) \mathfrak{C}_{i}^{(s)} S_{1}(n-i-l, m) D_{l}^{(k)}\left(a_{1}, \ldots, a_{r}\right) .
\end{aligned}
$$

Thus, we get the identity (33).

\section{Competing interests}

The authors declare that they have no competing interests.

\section{Authors' contributions}

All authors contributed equally to the manuscript and typed, read, and approved the final manuscript.

\section{Author details}

${ }^{1}$ Department of Mathematics, Sogang University, Seoul, 121-742, Republic of Korea. ${ }^{2}$ Department of Mathematics, Kwangwoon University, Seoul, 139-701, Republic of Korea. ${ }^{3}$ Graduate School of Science and Technology, Hirosaki University, Hirosaki, 036-8561, Japan. ${ }^{4}$ Division of General Education, Kwangwoon University, Seoul, 139-701, Republic of Korea.

\section{Acknowledgements}

This work was supported by the National Research Foundation of Korea (NRF) grant funded by the Korean government (MOE) (No. 2012R1A1A2003786). The second author was supported by Kwangwoon University in 2014. 


\section{References}

1. Kim, DS, Kim, T: Higher-order Cauchy of the second kind and poly-Cauchy of the second kind mixed type polynomials. Ars Comb. 117 (2014, to appear)

2. Kim, T: An invariant $p$-adic integral associated with Daehee numbers. Integral Transforms Spec. Funct. 13, 65-69 (2002)

3. Kim, DS, Kim, T, Rim, S-H: On the associated sequence of special polynomials. Adv. Stud. Contemp. Math. (Kyungshang) 23, 355-366 (2013)

4. Ozden, H, Cangul, IN, Simsek, Y: Remarks on q-Bernoulli numbers associated with Daehee numbers. Adv. Stud. Contemp. Math. (Kyungshang) 18, 41-48 (2009)

5. Kamano, K, Komatsu, T: Poly-Cauchy polynomials. Mosc. J. Comb. Number Theory 3, 183-209 (2013)

6. Simsek, Y, Kim, T, Pyung, I-S: Barnes' type multiple Changhee q-zeta functions. Adv. Stud. Contemp. Math. 10(2), 121-129 (2005)

7. Roman, S: The Umbral Calculus. Dover, New York (2005)

8. Dolgy, DV, Kim, DS, Kim, T, Komatsu, T, Lee, S-H: Barnes' multiple Bernoulli and poly-Bernoulli mixed-type polynomials. J. Comput. Anal. Appl. (to appear)

9. Ryoo, CS, Song, H, Agarwal, RP: On the roots of the q-analogue of Euler-Barnes' polynomia. Adv. Stud. Contemp. Math. 9, 153-163 (2004)

10. Comtet, L: Advanced Combinatorics. Reidel, Dordrecht (1974)

11. Kim, DS, Kim, T: Some identities of Frobenius-Euler polynomials arising from umbral calculus. Adv. Differ. Equ. 2012, 196 (2012)

12. Carlitz, L: A note on Bernoulli and Euler polynomials of the second kind. Scr. Math. 25, 323-330 (1961)

13. Liang, $\mathrm{H}$, Wuyungaowa: Identities involving generalized harmonic numbers and other special combinatorial sequences. J. Integer Seq. 15, Article 12.9.6 (2012)

10.1186/1687-1847-2014-140

Cite this article as: Kim et al.: Barnes-type Daehee of the first kind and poly-Cauchy of the first kind mixed-type polynomials. Advances in Difference Equations 2014, 2014:140

\section{Submit your manuscript to a SpringerOpen ${ }^{\ominus}$ journal and benefit from:}

- Convenient online submission

- Rigorous peer review

Immediate publication on acceptance

Open access: articles freely available online

- High visibility within the field

- Retaining the copyright to your article 